\title{
A TWO-MINUTE ASSAY FOR ELECTRONIC QUANTIFICATION OF ANTIBODIES IN SALIVA ENABLED THROUGH MULTI-FREQUENCY IMPEDANCE CYTOMETRY AND MACHINE LEARNING ANALYSIS
}

\author{
Zhongtian Lin ${ }^{l}$, Jianye Sui ${ }^{l}$, Pengfei Xie ${ }^{l}$, Karan Ahuja ${ }^{l}$ and Mehdi Javanmard ${ }^{l}$ \\ ${ }^{1}$ Department of Electrical and Computer Engineering, Rutgers University, USA
}

\begin{abstract}
At present, the use of saliva as a diagnostic fluid is more and more common due to its ability to monitor health status, onset and progression of disease and treatment progress in a noninvasive way. Rapid detection of antibodies in saliva can enable point-of-care diagnosis for auto-immune diseases like sepsis. For the first time, to the best of our knowledge, we have developed an electronic assay using impedance cytometry in conjunction with supervised machine learning, capable of quantifying immunoglobulins in saliva within two minutes.
\end{abstract}

\section{INTRODUCTION}

At present, the use of saliva as a diagnostic fluid is more and more common due to its ability to monitor health status, onset and progression of disease and treatment progress in a noninvasive way. [1] The state of various diseases can be diagnosed by monitoring the level of certain protein biomarkers in saliva. For example, in the case of chronic diseases like hepatitis B virus (HBV) and hepatitis $\mathrm{C}$ virus (HCV), HBV and HCV DNAs, antibodies exist in the saliva having a correlation with their levels in blood samples. [2-5] Auto-immune diseases like sepsis also have related biomarkers in saliva. [6] However, current technologies for performing biomarker assays usually involve bulky instrumentation with optical technologies like sandwich ELISA or protein microarrays. Light weight, highly sensitive and inexpensive ultra-compact platforms for point of care diagnosis are needed for those diseases.

Electrical impedance based technology are one of the most promising solutions to build portable platforms. Previously, we demonstrated detection of protein biomarkers in bioactivated microchannel [7] and used protein capture beads and impedance cytometry for detection of proteins with low abundance. [8] We also demonstrated another method for detection of protein based on bead aggregate sizing. [9] Besides, Carbonaro et. al. demonstrated a method for label free biomarker detection based on size changes of beads due to biomarker binding. [10] Tien-Li et. al. described a detection method for protein based on nanogap electrodes. [11] Tami et. al. demonstrated a frequency dependent electrochemical impedance spectroscopy to detect protein biomarkers. [12] And Gengfeng et. al. demonstrated a label-free multiplexed electrical detection of cancer makers using silicon-nanowire field-effect sensor arrays. [13] Jay et. al. demonstrated a label-free electrical detection of cardiac biomarker in real time using silicon nanowire sensor arrays. [14] For the first time, to the best of our knowledge, we have developed an electronic assay using impedance cytometry in conjunction with supervised machine learning, capable of quantifying immnunoglobulins in saliva within two minutes.

Figure 1 illustrates the assay steps and device operation. The assay works by using magnetic anti-IgG (primary antibody) coated beads to capture soluble IgG (target antigen) molecules in saliva. Anti-IgG (detector antibody) is then added to the magnetic beads to further increase the effective radius of the bead-protein complex. The beads flow through an impedance cytometer that probes the impedance of the beads at eight frequencies. The use of multi-frequency electrical impedance cytometry and machine learning technique allows for electronically determining the level to

which beads have captured target IgG molecules in saliva. We independently confirmed that binding properly occurred by fluorescently tagging (FITC) the detector anti-IgG molecules and imaged optically.

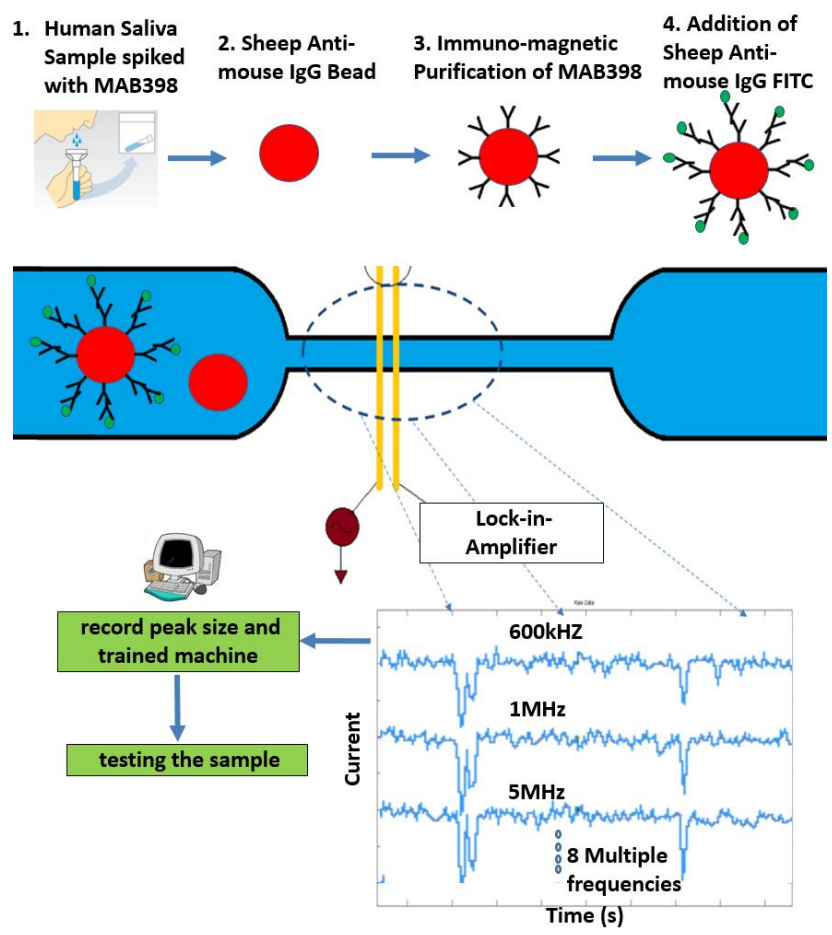

Figure 1. Schematic of biochip. Presence of $\operatorname{Ig} G$ in saliva sample results in beads binding to $\operatorname{Ig} G$ which enables the labeling of FITC markers to the beads. Multi-Frequency Impedance based sizing and machine learning technique were used for differentiating between FITC coated beads and unbound beads.

\section{THEORY}

The particle inside the channel with the electrode/electrolyte interface can be modelled using the circuit shown in Figure 2. We assumed an ideal polarizable electrode model without charge transfer resistance at the interface as we used gold as our electrode material, which is inert. The model involves two Debye layer capacitances $(\mathrm{Cdl})$ at each electrode in series with the solution resistance in series with an impedance network representing beads. We consider beads consists of a capacitance $(\mathrm{Cb})$ at its surface in series with resistance $(\mathrm{Rb})$.

$$
Z=\frac{2}{j \omega C_{d l}}+R_{\text {solution }}+\frac{1}{j \omega C_{\text {bead }}}+R_{\text {baed }}
$$

The impedance of the beads depends on the bead size and conductivity and dielectric permittivity at the bead surface. The binding of IgG modulates these properties at small levels, which makes it difficult to be detected by normal impedance cytometry at one or two frequencies Thus multi-frequency impedance cytometry and machine learning analysis are used in order to detect the 
differences.

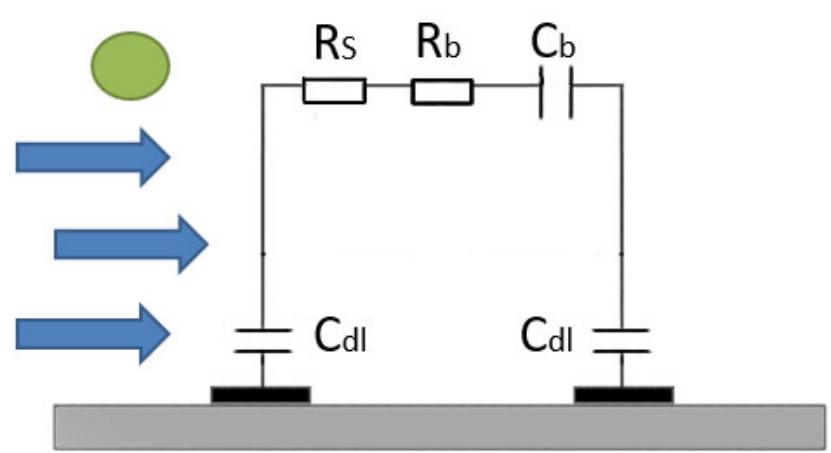

Figure 2. The equivalent circuit model for a two electrode pair system with a bead suspended in buffer.

\section{EXPERIMENTAL}

\section{Electrodes Fabrication}

The microfabricated biochip (Fig $3 a$ and Fig 3b) consists of two gold microelectrodes on a glass substrate with the PDMS (Polydimethyl Siloxane) channel above it. To fabricate the gold electrodes, standard photolithography was applied on a 3 inch fused silica wafer. The whole process includes photo-pattering resist on a fused silica wafer, performing electron beam metal evaporation and lift off process. The photo-pattering process consists of cleaning the wafer, spin coating photoresist, photoresist soft bake, ultra-violet light exposure through a four inch chromium mask, resist development, followed by a hard bake. $5 \mathrm{~nm}$ of chromium was deposited on the wafer via electron beam evaporation for enhancing the adhesion of the $100 \mathrm{~nm}$ gold layer to the wafer which was deposited after using the same methodology. Lift off was performed last using ultrasonic cleaner in acetone.

\section{Microfluidic Channel Fabrication}

The micro-channel starts from a $300 \mu \mathrm{m}$ wide and $20 \mu \mathrm{m}$ high channel part and tapers down to a $30 \mu \mathrm{m}$ wide and $20 \mu \mathrm{m}$ high sensing pore. The smaller cross sectional area of the sensing pore improves the focusing of particles above sensor and also increase electrical sensitivity to the extent where $2.8 \mu \mathrm{m}$ anti-IgG beads and IgG bind $2.8 \mu \mathrm{m}$ anti- IgG beads can be differentiated. The spacing between the two electrodes is $30 \mu \mathrm{m}$ and the width of each electrode is $20 \mu \mathrm{m}$. The micro-channel was made in PDMS from a master mold fabricated using soft lithography. The fabrication process consists of cleaning the wafer, spin coating, soft bake, photo-pattering an inverse feature onto a three inch silica wafer, UV light exposure, development and hard bake. To make PDMS channels, pre-polymer and curing agent were mixed at the ratio of 10 to 1 and poured onto the master mold. After curing the PDMS for about 1 hour, we peeled it off, cut the channels out and punched holes to make inlets and outlets. At last, the microfluidic channel was aligned to the electrode substrate and bonded onto the glass wafer covalently by treating both substrates with oxygen plasma.

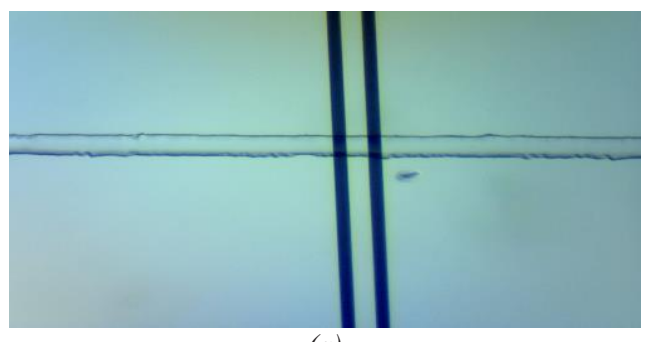

(a)

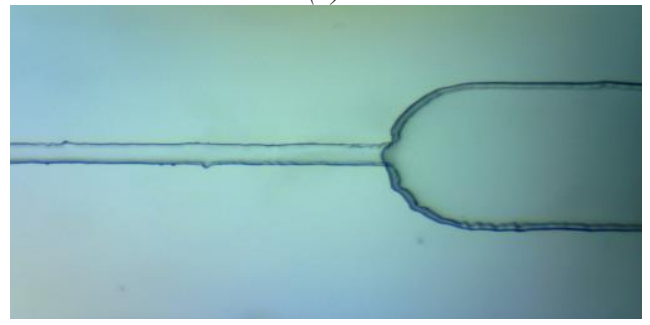

(b)

Figure 3. (a) Microfabricated electrodes (b) Entrance of micropore

\section{Measurements}

In order to make the beads flow without the help of a pump to minimize the influences from external interference, we first performed plasma treatment on the channel to make it hydrophilic and filled it with PBS to keep its hydrophilicity. Due to the pressure differences between the inlet and outlet, which was generated by the differences in fluid volume in the well, the beads were then injected into the inlet of the biosensor and directed to the multi-frequency impedance sensor which was connected to a lock-in amplifier (Zurich Instrument HF2 series). The input AC voltage was $400 \mathrm{mV}$ peak-to-peak and the gain was $1 \mathrm{kV} / \mathrm{Amp}$. As particles passed over the electrodes, impedance responses at 8 discrete frequencies from $600 \mathrm{kHz}$ to $24 \mathrm{MHz}$ were captured simultaneously. A metal box was used to electrically insulate the sensor from external interference during testing. The recorded data was then processed using a custom-written Matlab code which performed detrending, denoising, peak finding, and differentiating the beads bound to IgG from the normal beads with the help of machine learning. At the same time, in order to confirm the binding both electrically and optically, we tagged the beads with fluorescent markers and observed the beads under a fluorescent microscope immediately after the assay.

\section{Sample Preparation}

To demonstrate proof-of-concept, saliva samples were spiked with mouse IgG at concentrations of $1667 \mathrm{nM}, 167 \mathrm{nM}$ and $17 \mathrm{nM}$. We used $2.8 \mu \mathrm{m}$ paramagnetic Dynal beads (Life Technologies, Carlsbad, CA) coated with anti-mouse IgG antibodies to capture the mouse IgG in the saliva sample. The protocol for performing the 2 min assay went as follows: Sheep anti-mouse $\operatorname{IgG}$ beads were washed three times in PBS $0.1 \%$ BSA. The beads were then mixed with mouse IgG spiked saliva sample off chip in the epindorph tube and rotate for $2 \mathrm{~min}$. In order to confirm that the binding properly occurred, we then washed the beads three times with PBS $0.1 \%$ BSA and mixed with FITC tagged sheep anti-mouse IgG. Beads were washed with PBS to ensure all the unbound antibodies were washed away before the electrical testing.

\section{Machine learning algorithm}

To improve classification accuracy and be able to detect the differences between beads that captured no analyte and beads that captured IgG, a support vector machine (SVM) machine learning 
algorithm is employed, where peak amplitudes at 8 frequencies are used as features in the feature vector. Classification of different particles improves effectively in high-dimensional space. The SVM model gets trained using part of the data, and the rest of the data are used for testing. Comparing the prediction with the true category, the accuracy was calculated.

\section{RESULTS AND DISCUSSION}

Experiments were performed both optically and electrically at multiple IgG concentrations to verify the viability and repeatability of the 2 minute assay. Figure 4 a shows the fluorescent image of the positive assay where beads were incubated for two minutes in an IgG positive saliva sample and Fig. $2 \mathrm{~d}$ for the control assay where beads incubated for the same time in an IgG negative saliva sample. The positive assay shows strong florescent signal compared to the black background negative control assay as a result of specific anti-IgG fluorescently tagging. The image confirmed that the $\mathrm{IgG}$ bind to the beads.
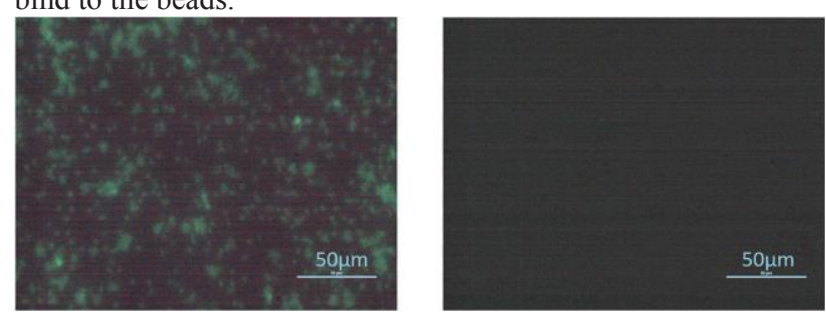

Figure 4. (a) IgG positive beads (anti-IgG detector antibody FITC tagged) (b) IgG negative beads.

As for the electrical testing, when the sample was injected onto the biosensor using pressure driven flow, particles passed over the electrodes, which were connected to an $\mathrm{AC}$ voltage source and a multi-frequency lock-in amplifier, a frequency dependent change in current is exhibited as showed in raw data (Fig 5).

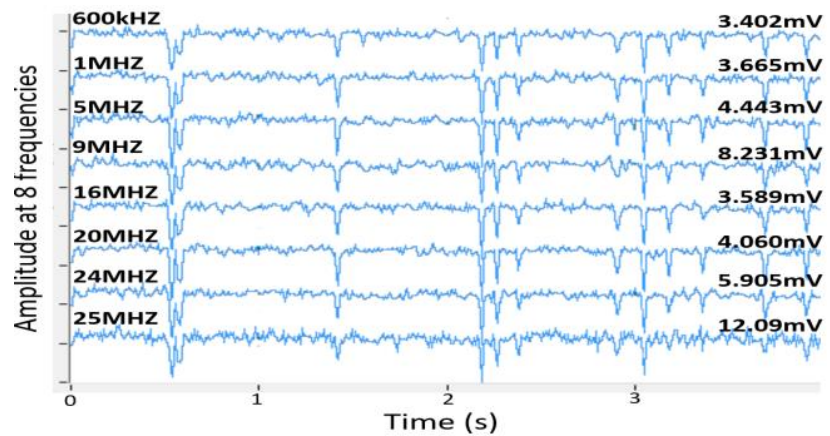

Figure 5. Raw data of beads passing through impedance cytometer at multiple frequencies. The frequencies were $600 \mathrm{~K}, 1 \mathrm{M}, 5 \mathrm{M}, 9 \mathrm{M}$, $16 \mathrm{M}, 20 \mathrm{M}, 24 \mathrm{M}, 25 \mathrm{MHz}$. The amplitudes of the Input AC voltage were $400 \mathrm{mV}$.

The amplitude of the current peak at different frequencies depends on bead size and conductivity and dielectric permittivity at the bead surface. Single and even dual frequency analysis alone makes it difficult to distinguish different bead types from each other as shown in the scatter plot for voltage peak intensity at $600 \mathrm{kHz}$ and $24 \mathrm{MHz}$ (Fig 6) or at $1 \mathrm{Mhz}$ and 20Mhz (Fig 7).

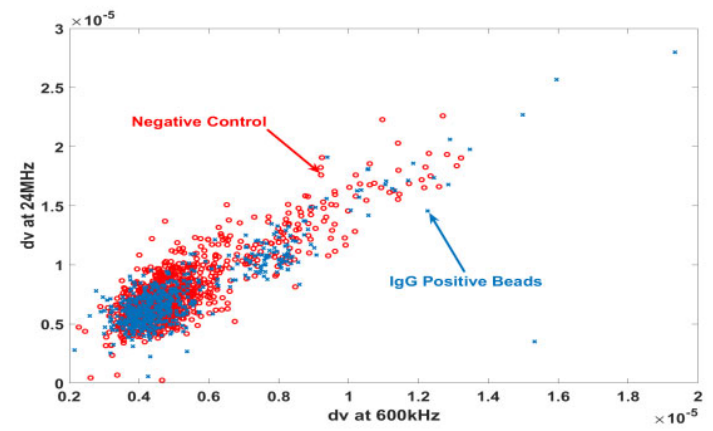

Figure 6. Scatter plot for voltage peak intensities at $600 \mathrm{kHz}$ and $\mathrm{dv}$ at $24 \mathrm{MHz}$.

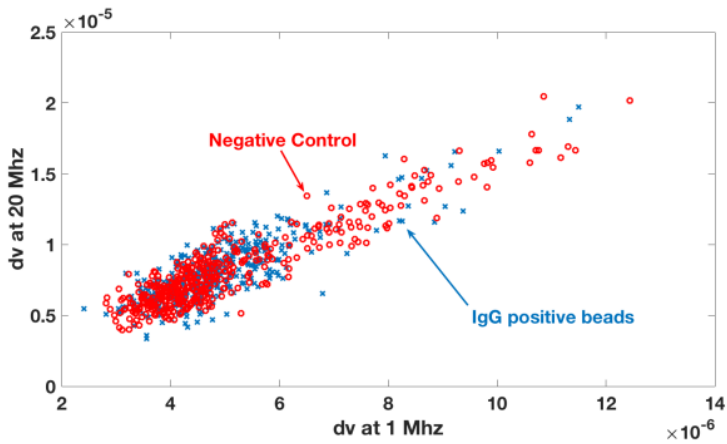

Figure 7. Scatter plot for voltage peak intensities at $1 \mathrm{MHz}$ and $d v$ at $20 \mathrm{MHz}$.

The use of eight frequencies, however, in conjunction with machine learning makes bead differentiation and thus target $\mathrm{IgG}$ quantification feasible. We trained the SVM (Support Vector Machine) model with peak intensities from IgG positive and IgG negative data at eight frequencies, and then verified this model on test data from different samples and determined algorithm classification accuracy for IgG positive and IgG negative beads. As the concentration of target IgG in saliva decreases, SVM classification accuracy decreases. We used this classification accuracy as a metric to quantify IgG levels (Fig. 8). We formulated a biomarker quantification score (Eq. 2 ) to provide a self-calibrated method of quantifying biomarker levels. Figure 5 shows a biomarker quantification score dynamic range of 3 orders of magnitude.

$$
\text { Biomarker Quantification Score }=\frac{\text { SVM Accuracy }-50}{50}
$$




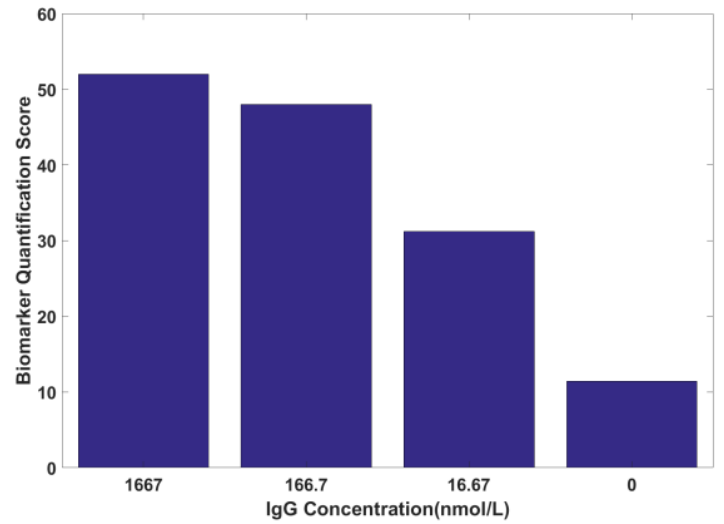

Figure 8. Bar graph of Biomarker Quantification Score to $\operatorname{Ig} G$ concentrations at 0 to $1667 \mathrm{nM}$;

\section{CONCLUSIONS}

In conclusion, our experimental results show that through the combination of multi-frequency microfluidic impedance cytometry and supervised machine learning, that we are able to qualify immunoglobulins in saliva within two minutes. Though in this work, IgG was used as the biomarker for testing in saliva samples, the method can be performed in a wide variety of proteins as long as a comparable high affinity antibody pair is available. The work shown here has potential to be developed into an integrated biochip to rapidly quantify certain biomarker levels in complex samples like saliva and urine. Our future work will be focused on increasing the sensitivity for this assay from nanoMolar to picoMolar to make it comparable to the gold standard ELISA method and validate this method in a variety of proteins.

\section{ACKNOWLEDGEMENTS}

This project was funded by PhRMA Foundation Starter Grant for Early Faculty. This research was sponsored by an anonymous corporation. The devices were fabricated in the Microelectronics Research Laboratory (MERL) in Rutgers University.

\section{REFERENCES}

[1] Streckfus, C. F., and L. R. Bigler. "Saliva as a diagnostic fluid." Oral diseases 8, no. 2 (2002): 69-76.

[2] Yoshizawa, Janice M., Christopher A. Schafer, Jason J. Schafer, James J. Farrell, Bruce J. Paster, and David TW Wong. "Salivary biomarkers: toward future clinical and diagnostic utilities." Clinical microbiology reviews 26, no. 4 (2013): 781-791.

[3] Chen, Lizhang, Fuqiang Liu, Xuegong Fan, Jianming Gao, Nengzhi Chen, Tom Wong, Jun $\mathrm{Wu}$, and Shi Wu Wen. "Detection of hepatitis B surface antigen, hepatitis B core antigen, and hepatitis B virus DNA in parotid tissues." International Journal of Infectious Diseases 13, no. 1 (2009): 20-23.

[4] Gonçalves, Patrícia L., Carla B. Cunha, Solange CU Busek, Guilherme C. Oliveira, Rodrigo Ribeiro-Rodrigues, and Fausto EL Pereira. "Detection of hepatitis C virus RNA in saliva samples from patients with seric anti-HCV antibodies." Brazilian Journal of Infectious Diseases 9, no. 1 (2005): 28-34.

[5] González, V., E. Martró, C. Folch, A. Esteve, L. Matas, A. Montoliu, J. R. Grifols et al. "Detection of hepatitis C virus antibodies in oral fluid specimens for prevalence studies." European Journal of Clinical Microbiology \& Infectious Diseases 27, no. 2 (2008): 121-126.
[6] Cho, Sung-Yeon, and Jung-Hyun Choi. "Biomarkers of sepsis." Infection \& chemotherapy 46, no. 1 (2014): 1-12.

[7] Javanmard, Mehdi, Amirali H. Talasaz, Mohsen Nemat-Gorgani, Fabian Pease, Mostafa Ronaghi, and Ronald W. Davis. "Electrical detection of protein biomarkers using bioactivated microfluidic channels." Lab on a Chip 9, no. 10 (2009): 1429-1434.

[8] Mok, Janine, Michael N. Mindrinos, Ronald W. Davis, and Mehdi Javanmard. "Digital microfluidic assay for protein detection." Proceedings of the National Academy of Sciences 111, no. 6 (2014): 2110-2115.

[9] Lin, Z., X. Cao, P. Xie, M. Liu, and Mehdi Javanmard. "PicoMolar level detection of protein biomarkers based on electronic sizing of bead aggregates: theoretical and experimental considerations." Biomedical microdevices 17, no. 6 (2015): 119.

[10] Carbonaro, A., and L. L. Sohn. "A resistive-pulse sensor chip for multianalyte immunoassays." Lab on a Chip 5, no. 10 (2005): 1155-1160.

[11] Chang, Tien-Li, Chien-Ying Tsai, Chih-Chen Sun, Chun-Chi Chen, Long-Sheng Kuo, and Ping-Hei Chen. "Ultrasensitive electrical detection of protein using nanogap electrodes and nanoparticle-based DNA amplification." Biosensors and Bioelectronics 22, no. 12 (2007): 3139-3145.

[12] Lasseter, Tami L., Wei Cai, and Robert J. Hamers. "Frequency-dependent electrical detection of protein binding events." Analyst 129, no. 1 (2004): 3-8.

[13] Zheng, Gengfeng, Fernando Patolsky, Yi Cui, Wayne U. Wang, and Charles M. Lieber. "Multiplexed electrical detection of cancer markers with nanowire sensor arrays." Nature biotechnology 23, no. 10 (2005): 1294.

[14] Chua, Jay Huiyi, Ru-Ern Chee, Ajay Agarwal, She Mein Wong, and Guo-Jun Zhang. "Label-free electrical detection of cardiac biomarker with complementary metal-oxide semiconductor-compatible silicon nanowire sensor arrays." Analytical chemistry 81, no. 15 (2009): 6266-6271.

\section{CONTACT}

*Mehdi Javanmard; mehdi.javanmard@rutgers.edu 